\title{
Distributed Physical Sensors Network for the Protection of Critical Infrastractures Against Physical Attacks
}

\author{
Conference Paper · January 2016
}

DOI: 10.5220/0006017601390150

CITATIONS

0

15 authors, including:
READS

66

\section{Christian Callegari}

Consorzio Nazionale Interuniversitario per le... 102 PUBLICATIONS 310 CITATIONS

SEE PROFILE

\section{Piotr Samczynski}

Warsaw University of Technology

133 PUBLICATIONS 362 CITATIONS

SEE PROFILE
Fabrizio Berizzi

Università di Pisa

187 PUBLICATIONS $\quad 1,636$ CITATIONS

SEE PROFILE

\section{Krzysztof Kulpa}

Warsaw University of Technology

321 PUBLICATIONS 1,147 CITATIONS

SEE PROFILE

Some of the authors of this publication are also working on these related projects:

Project

Project

Multitech SeCurity system for intercOnnected space control groUnd staTions-SCOUT project View project

uSAR radar - Poland View project 


\title{
Distributed Physical Sensors Network for the Protection of Critical Infrastractures Against Physical Attacks
}

\author{
M. P. Jarabo-Amores ${ }^{1}$, M. Rosa-Zurera ${ }^{1}$, D. de la Mata-Moya ${ }^{1}$, A. Capria ${ }^{2}$, A. L. Saverino ${ }^{2}$, \\ C. Callegari ${ }^{2}$, F. Berizzi ${ }^{2,3}$, P. Samczyński ${ }^{4}$, K. Kulpa ${ }^{4}$, M. Ummenhofer ${ }^{5}$, H. Kuschel ${ }^{5}$, A. Meta ${ }^{6}$, \\ S. Placidi ${ }^{6}$, K. Lukin ${ }^{7}$ and G. D'Amore ${ }^{8}$ \\ ${ }^{I}$ Signal Theory and Communications Department, Escuela Politécnica Superior, Universidad de Alcalá, \\ Ctra. Madrid-Barcelona, Alcalá de Henares, Spain \\ ${ }^{2}$ RaSS National Laboratory, National Inter-University Consortium for Telecommunications (CNIT), Pisa, Italy \\ ${ }^{3}$ Department of Information Engineering, University of Pisa, Pisa, Italy \\ ${ }^{4}$ Institute of Electronic Systems, Warsaw University of Technology, Warsaw, Poland \\ ${ }^{5}$ Fraunhofer Institute for High Energy Physics and Radar Techniques FHR, Fraunhofer Institute, Wachtberg, Germany \\ ${ }^{6}$ MetaSensing BV, Noordwijk, The Netherlands \\ ${ }^{7}$ LNDES, IRE NASU, Kharkiv, Ukraine \\ ${ }^{8}$ Vitrociset, Rome, Italy
}

\begin{abstract}
Keywords: $\quad$ SCOUT, Passive Radar, Noise Radar, RFID, Infrared Camera, Data Fusion, Target Classification, Data Link, Critical Infrastructure.

Abstract: The SCOUT project is based on the use of multiple innovative and low impact technologies for the protection of space control ground stations and the satellite links against physical and cyber-attacks, and for intelligent reconfiguration of the ground station network (including the ground node of the satellite link) in the case that one or more nodes fail. The SCOUT sub-system devoted to physical attacks protection, SENSNET, is presented. It is designed as a network of sensor networks that combines DAB and DVB-T based passive radar, noise radar, Ku-band radar, infrared cameras, and RFID technologies. The problem of data link architecture is addressed and the proposed solution described.
\end{abstract}

\section{INTRODUCTION}

Protection of critical buildings, plants and infrastructure is one of most important issues for the European Union (EU) community. Critical infrastructures consist of those physical and information technology facilities, networks, services and assets which, if disrupted or destroyed, would have a serious impact on the health, safety, security or economic well-being of citizens or prevent the effective functioning of governments in the Member States. Critical infrastructures extend across many sectors of the economy, including banking and finance, transport and distribution, energy, utilities, health, food supply and communications, as well as key government services (COM,2004).

The Multitech SeCurity system for intercOnnected space control groUnd staTions,
SCOUT, project is a solution for the Topic SEC2013.2.2-5: Security of ground based infrastructure and assets operating space systems (EU, 2013).

The SCOUT project is based on the use of multiple innovative and low impact technologies for the protection of space control ground stations and the satellite links against physical and cyber-attacks, and for intelligent reconfiguration of the ground station network in the case that one or more nodes fail.

The SCOUT system implements the three main security functionalities by three different subsystems controlled and governed by a centralized Main Control Unit (MCU), Figure 1:

- SENSNET for physical attack detection and protection.

- CYBERSENS for cyber-attack detection and countermeasures. 
- RECOVER for automatic restoration and intelligence reconfiguration of the space control ground station network, in the case of fault of one of the nodes.

Risk assessment tools drive both, design and system functionality.

This paper focusses on the SENSNET system and the data link architecture required for the operation, control and maintenance of the different sensor networks that belongs to it.

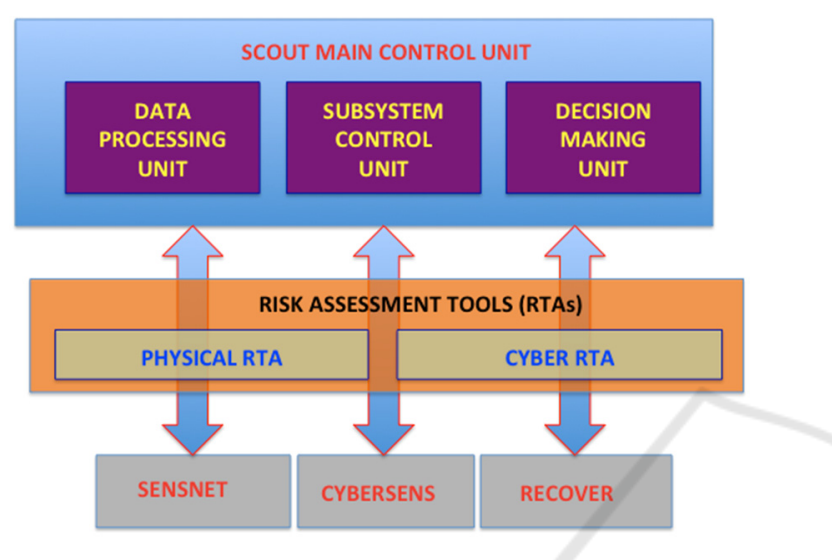

Figure 1: SCOUT system concept.

\section{PHYSICAL-SECURITY SENSOR NETWORK}

\subsection{Problem Formulation}

Nowadays, physical security systems can be classified into physical-based and remote sensingbased solutions:

- Physical-based systems are composed of sensors that detect the intruders when they physically come into contact with the sensor, or when they are in its near proximity.

They usually require a high number of sensors (wire, tubes, cables, vibration sensor) distributed along the fence or in the surrounding perimeter area; volumetric surveillance is not provided; installation and maintenance costs are not negligible.

- In remote sensing based systems, the intruder is detected far from the sensor, which is typically positioned in a remote site with respect to the surveillance area.

Two main categories of remote sensing based systems can be distinguished:

1. Systems operating in the optical and infrared bands: video motion detection sensors, CCTV surveillance systems and infrared detection units. Their main characteristics are the following: performances are heavily affected by overgrown vegetation, fog, heavy rain, snow, sand, storm, animals, debris, and movement of mounting posts that can be subjected to external manipulation; air intruder surveillance is not contemplated; limits for large area surveillance; low costs.

2. Remote sensing security systems operating in the microwave region: microwave movement detectors, radar detection systems. Their main characteristics are: large area volumetric protection, including air targets if the radar has a high elevation beamwidth antenna; all weather/all day operating; detection of stationary and moving targets, emission of power (e.m. pollution, high costs for the presence of the transmitter, safety of humans subjected to very high frequency e.m. radiations, high probability of intercept by Electronic Support Measurement (ESM) system); possibility of deactivation through the use of jammers; microwave systems based on electric field flooding of the surveillance area are strongly affected by other electrical fields interferences.

From the above analysis, we can conclude that remote sensing security systems based on radar technology are really promising and reliable solutions. Nevertheless, the radar has the main drawback that it emits power, and can be easily intercepted and deactivated by jammers.

Security surveillance is usually limited to a building, and the 'attack' can be recognized only once it has started. For the reasons above, the physical security of this critical infrastructure can be greatly improved by a system that can:

- extend the monitored area to the entire building perimeter, even beyond the fence for terrestrial and airborne attacks.

- anticipate all critical situations (early warning).

- preserve the privacy of people not involved in the buildings normal interactions (limit the use of image-based systems), since the refinery is a crowded place.

- make use of multiple technologies which are fault tolerant with respect to light and weather conditions and that guarantees a suitable level of redundancy.

- intelligently distinguish between people, animals and inanimate objects.

- detect vehicles and obstacles which may hide dangerous people. 
- limit the number of false alarms by properly filtering only the important information to the control room operator, in order to preserve the systems efficiency.

- reduce the installation and maintenance costs.

- reduce the electromagnetic pollution (the system must not be invasive).

\subsection{SENSNET Subsystem}

The main functional block of the physical sensor network SENSNET is depicted in Figure 2:

- A distributed sensor network interacts with the surveillance area to acquire information about the presence of potential physical threats, and perform a first level detection.

- The multi-sensor detection and tracking stage applies data fusion techniques to improve detection capabilities of the overall system and perform target tracking.

- Detected targets data will be applied to a classification stage to discriminate between aerial targets, terrestrial targets and living things.

- Classifier outputs will be delivered to the MCU. Design drivers combined those defined in section

2.1. and the following:

- Detection of stationary and moving targets.

- Capability of identifying authorized people and vehicles.

- Reduce the electromagnetic pollution and avoid the requirement of band allocation: (Royal Decree, 2001), (ECA Table, 2014), (Mazar, 2014).

- Low probability of intercept (LPI).

- Reconfigurable, modular, high performance and robust solutions.

- Low power consumption.

- Use of data fusion techniques to improve detection, tracking and classification performances.

\subsubsection{Distributed Physical Sensor Network}

The distributed physical sensor network includes systems operating in the microwave and infrared regions, and Radio Frequency Identification, RFID, systems.

Microwave systems selection was guided by the following goals:

- Null or very low power emission.

- Fault tolerant with respect to light and weather conditions.
- Capable of performing volumetric protection (ground and aerial targets), early warning, and detection of stationary and moving targets.

The proposed solution includes Passive Bistatic Radars (PBR); noise radars, and radar imaging sensors for target classification.

Infrared cameras provide target images at high spatial resolution, also in dark conditions, which are useful for target classification.

Microwave and infrared imaging system guarantee the preservation of people privacy.

RFID mounted on friendly people and vehicles will be used to identify authorized people and vehicles.

The above sensors are equipped with an own detector, whose outputs will be a first level of detection (pre-detections).

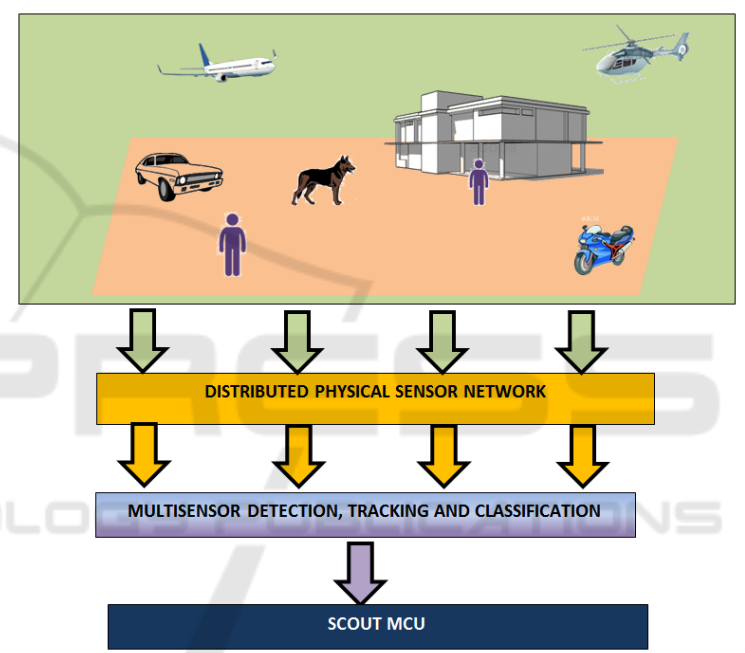

Figure 2: Physical sensor network functional block diagram.

\subsubsection{Multi-sensor Detection, Tracking and Classification}

A multi-sensor detector based on data fusion techniques refines the single sensor plots and provides the final detections with higher performance in terms of probability of detection, and probability of false alarms, by properly filtering only the important information to the control room operator, in order to preserve the systems efficiency.

A first level classifier provides a preliminary classification of unknown targets in three classes: aerial vehicle, terrestrial vehicle, and possible humans. Known people detected and tracked by the RFID system are discarded.

Targets classified as possible humans will be illuminated by infrared and microwave imaging 
systems to generate images that will be driven to the second level classifier in charge of classifying into three classes: humans, animals, and others.

\section{SYSTEM REQUIREMENTS}

SENSNET operative requirements are summarized in Table 1. The coverage area is structured in four sub-areas (Figure 3):

- The aerial early alert area defines a ring centred on the facility central point, and extends from $10 \mathrm{~km}$ to $20 \mathrm{~km}$. The objective is the early detection of low altitude aerial targets approaching the facility.

- Targets to be sought are big-medium commercial airplanes and small aircrafts (CESSNA type).

- The higher resolution aerial surveillance area defines a circle centred in the central point of the facility with a radius of $10 \mathrm{~km}$.

- Targets of interest are small aircrafts, medium size ( $>5 \mathrm{~m}$ of wingspan) drones, and ultralight aircrafts with cockpit.

- External area close to the perimeter fence, defined by a radius $2-3 \mathrm{~km}$ bigger than the distance of outermost point of the perimeter fence from the central point of the facility. Targets to be sough include aerial ones (ultralight aircrafts or small drones), medium distance weapons (shoulder-launched rockets) and ground vehicles (trucks, vans or cars). Persons trying to climb or run through the fence will be also targets of interest.

Table 1: SENSNET operative requirements.

\begin{tabular}{|l|l|}
\hline OR-PHY-1 & \multicolumn{1}{|c|}{ Detection of aerial attacks } \\
\hline Description & $\begin{array}{l}\text { Detection of small aircrafts flying at low } \\
\text { altitudes }\end{array}$ \\
\hline OR-PHY-2 & $\begin{array}{l}\text { Preventive defensive measures against } \\
\text { short-medium distance weapons }\end{array}$ \\
\hline Description & $\begin{array}{l}\text { Detection of vehicles approaching the } \\
\text { facilities using non-conventional routes, } \\
\text { stopping in areas without parking facilities, } \\
\text { or with too high velocities. Detection of } \\
\text { shoulder-launched rockets }\end{array}$ \\
\hline OR-PHY-3 & $\begin{array}{l}\text { Surface movement monitoring inside } \\
\text { facilities perimeter }\end{array}$ \\
\hline Description & $\begin{array}{l}\text { Surface monitoring is required for } \\
\text { detecting authorized vehicles or people } \\
\text { that trying to access non-authorized areas }\end{array}$ \\
\hline
\end{tabular}

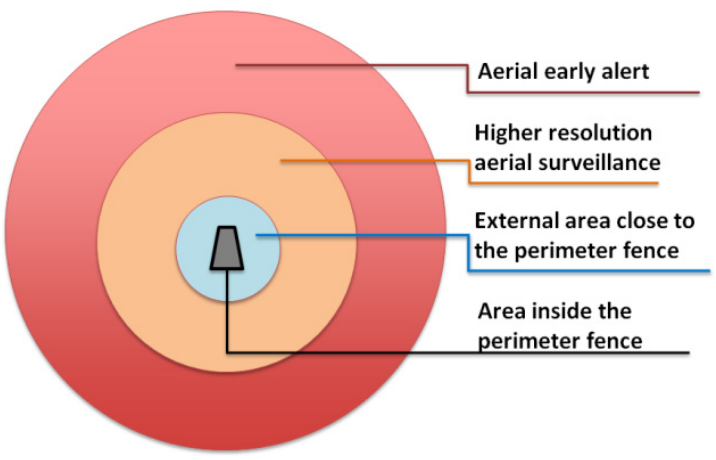

Figure 3: Coverage areas.

\section{SCENARIO DEFINITION}

The case study is defined on the Tracking \& Imaging Radar (TIRA) system site, located on a campus shared by the two Fraunhofer Institutes FHR and FKIE (Figure 4). A 3D CAD model was built for the analysis of shadowed areas (Figure 5).

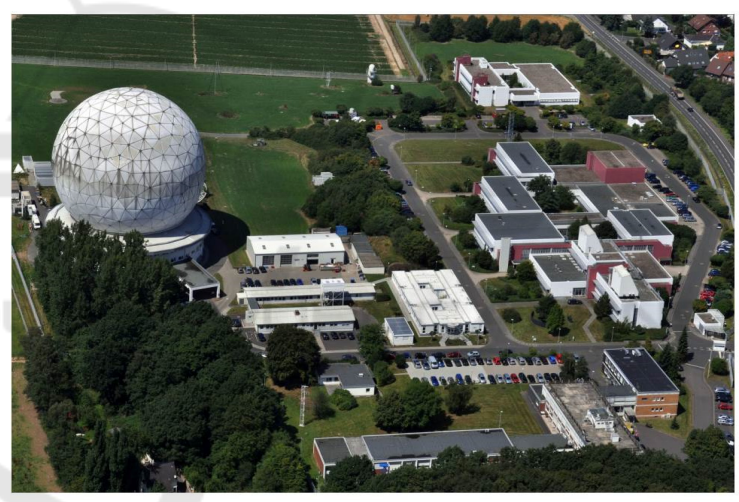

Figure 4: TIRA site.

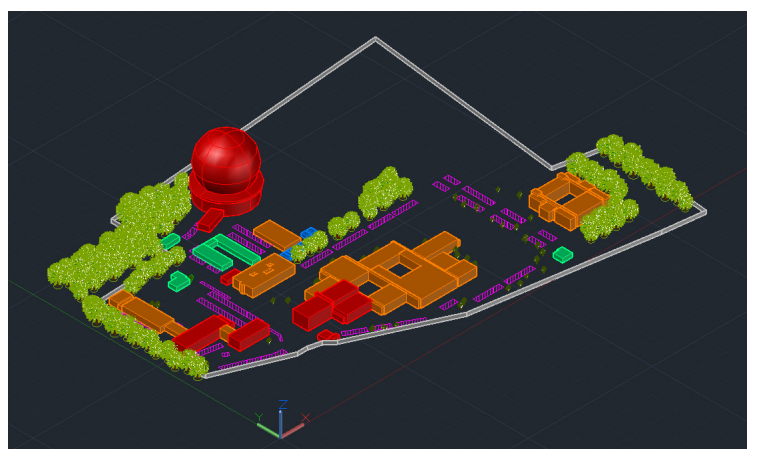

Figure 5: 3D CAD model of the TIRA site. Colours represent priority level: high priority (red), medium priority (orange), low priority (green), parking (pink). 


\section{SENSNET ARCHITECTURE}

SENSNET architecture is depicted in Figure 6. In the following subsections, SENSNET technologies are described.

\subsection{Passive Radar Network}

A Passive Bistatic Radar (PBR) can be defined as a set of radar techniques that use non-cooperative signals, such as broadcast, communications, radar, or radio-navigation signals as Illuminators of Opportunity (IoO), rather than a dedicated transmitter (IEEE, 2008). These systems are very attractive due to their low costs, their low probability of interception, and low power consumption. The basic system geometry is shown in Figure 7.

A dual channel reception system is required: a surveillance channel for targets acquisitions, and a reference one, for capturing the IoO signal. Target echoes signals will be correlated with Doppler shifted copies of the reference signal to generate the CrossAmbiguity Function, CAF, that will provide processing gain and the capability of estimating bistatic range and Doppler of the detected targets. Digital Audio Broadcasting (DAB) and Digital Video Broadcasting-Terrestrial (DVB-T) were selected as IoOs (Coleman, 2008), (Saini, 2005), (Conti, 2010), (Kulpa, 2011), (Gomez-del-Hoyo, 2015).

Available IoOs in the considered case of study are shown in Figure 8 and Figure 9.

The proposed passive radar node architecture is based on Software Defined Radar, SDR, principles (Figure 10). Antenna arrays are used due to the angular resolutions required by the targets to be sought, and the coverage requirements.

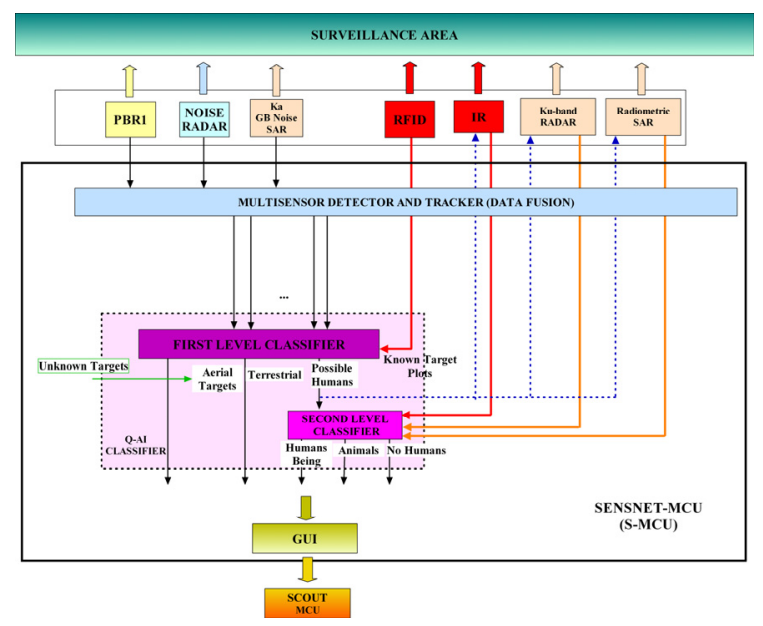

Figure 6: SENSNET general architecture.

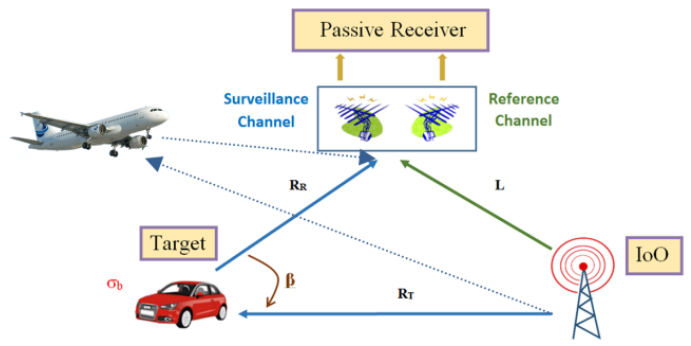

Figure 7: PBR geometry.

Sensor emplacement techniques will be applied together with the information of the available IoOs to design the final network architecture. Beamforming techniques will play a key role, allowing the generation of multiple radiation beams in order to fulfill the required instrumented angular coverage and azimuth resolutions. The first approach of passive radar network structure is shown in Figure 11 .

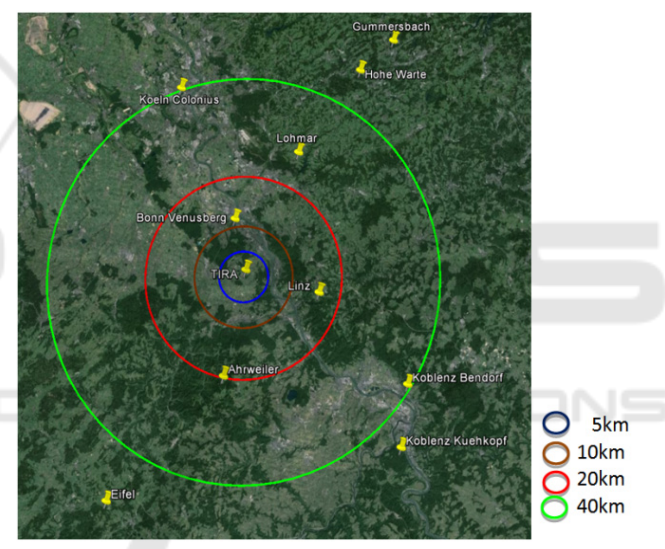

Figure 8: Available DVB-T IoOs.

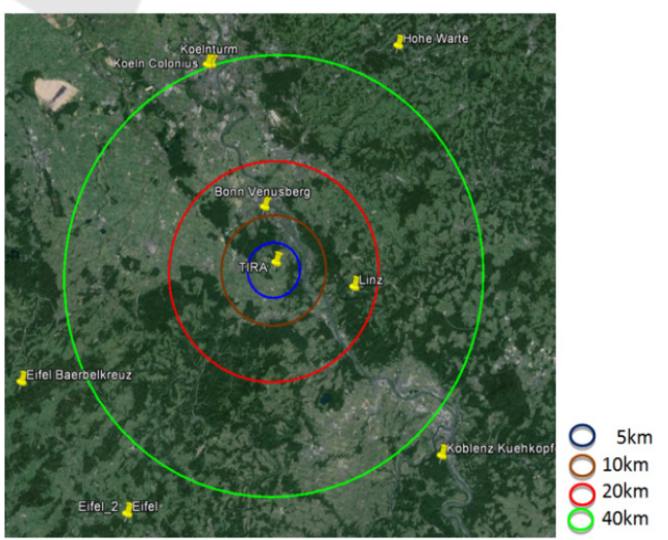

Figure 9: Available DAB IoOs. 


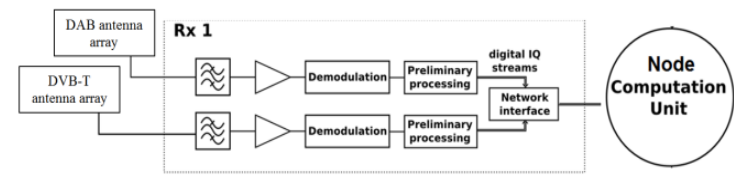

Figure 10: Basic scheme of a PBR node.

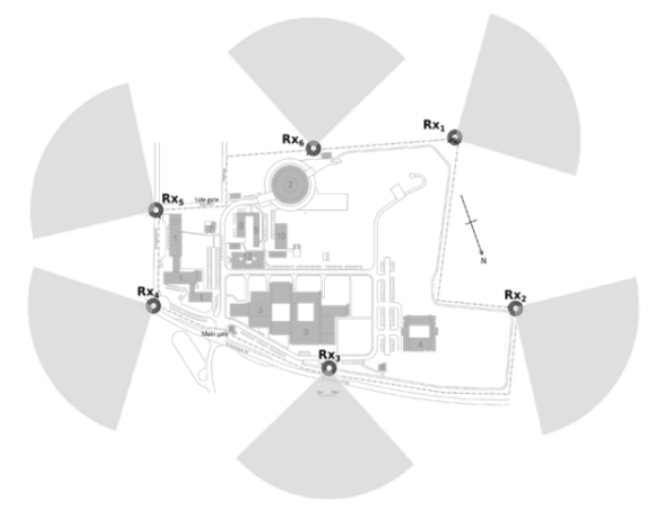

Figure 11: First approach of passive radar network architecture.

\subsection{Noise Radar}

Noise Radars (NR) use random noise, pseudorandom or chaotic waveforms as sounding signals, and coherent processing for target detection. These signals provide important properties: optimal coherent reception and high compression rate, independent control of velocity and range resolutions, no side lobes in Ambiguity Function, no range ambiguity for both Continuous Wave (CW) and pulse radar.

NR enables better performance in LPI, immunity against interferences and/or jamming, and better electromagnetic compatibility performance between different units and different types of radar sensors. Spectrum sharing problem may be easier solved using correlation properties of noise waveforms with wide enough power spectrum bandwidth. Two NR sensor networks are proposed:

- A C-band noise radar network for the detection of medium distance weapons (shoulder-launched rockets) and ground vehicles (trucks, vans or cars) in the external area close to the perimeter fence, and for complementing passive radars for the detection of ultralight aircrafts or small drones approaching the perimeter fence in the higher resolution aerial surveillance area (Malanowski, 2012), (Shelevytsky,2013).

- A Ka-band Ground Based Noise Waveform SAR (GB NW SAR) network for the detection of moving targets in the inside perimeter area, in combination with RFID sensors, and for complementing passive and C-band noise radars for the detection of the most challenging targets approaching the perimeter fence: ultralight aircrafts, small drones and persons (Lukin,2008), (Lukin,2005).

Figure 12 shows a possible Ka-band GB NW SAR sensor network configuration for the detection of objects inside the TIRA-site, and others approaching the main gates or other parts of the perimeter, being a complement of passive radar and C-band noise radar networks for the detection of ultralight aircrafts, small drones and persons approaching the perimeter fence.

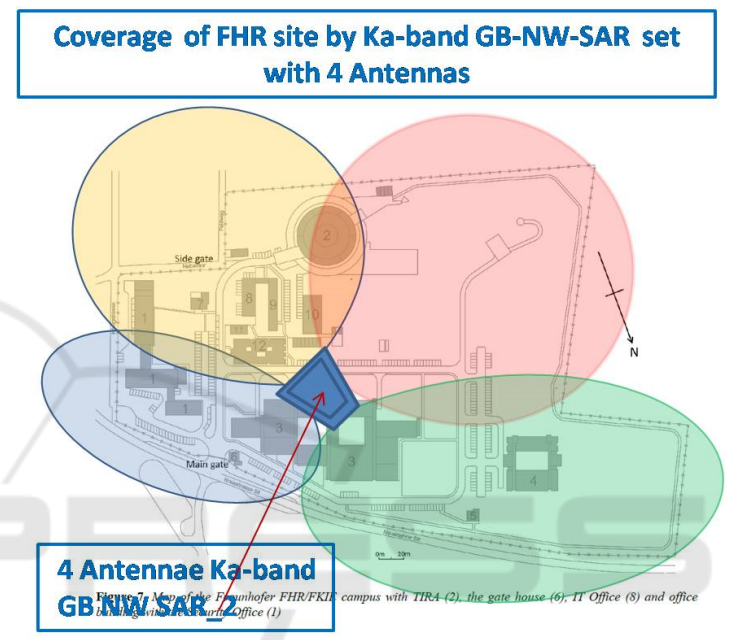

Figure 12: TIRA site coverage with GB NW SAR sensor network with four antennas.

\subsection{Imaging Sensors}

Two types of sensors are responsible of target images generation for classification purposes:

- A Ku-band system which operates in real aperture mode, and can provide high resolution radar data which can be used with Inverse SAR algorithms for the generation of the target's images (Metasensing. 2015), (Marotti, 2015).

- A network of Pan-Tilt-Zoom (PTZ) cameras, which are characterized by an angular Field of View, FoV, that can vary along the three dimensions: horizontal (pan), vertical (tilt), and depth (zoom). Midwave and Longwave cameras are used, because they do not require an illumination source.

\subsection{RFID Sensor Network}

The goal of the RFID sensors network is to monitor and track critical people and/or objects in the area 
inside the perimeter fence, to avoid that people can access an unauthorized area without proper permission (Vitrociset2012).

The data acquired though the sensors will be managed through a centralized server, connected to the overall SCOUT infrastructure via a proper link (Figure 13).

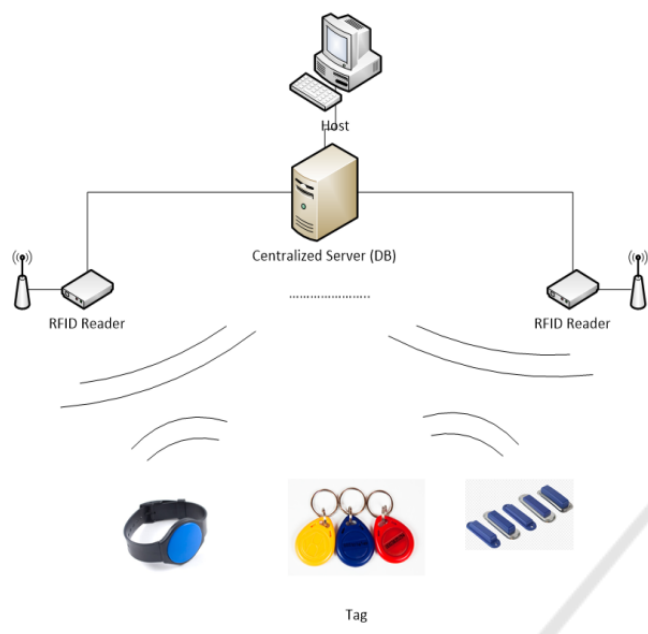

Figure 13: RFID Centralized Data Management.

The system is designed following a hybrid approach, based on the consideration that the localization will be divided in two main parts and that different RFID active transponders can be used:

- Outdoor (Figure 14) - the target's localization is made with estimation techniques (Received Signal Strength, Time Of Arrival, Time Difference Of Arrival, Received Signal Phase, Angle Of Arrival);

- Indoor (Figure 15) - the target's localization is made with proximity techniques based on a dense deployment of antenna.

The proposed architecture can be completely customized considering the user's needs and the Ground Station structure.

\section{DATA LINK ARCHITECTURE}

A basic communication point-to-multipoint topology is defined in Figure 16, assuming the SENSNET general architecture (Figure 6). Each subsystem is composed by a set of sensors distributed throughout the facility area that are connected to the MCU. In Figure 16, each sensor network is represented by a cloud, although, in each network, each node is connected to the MCU.

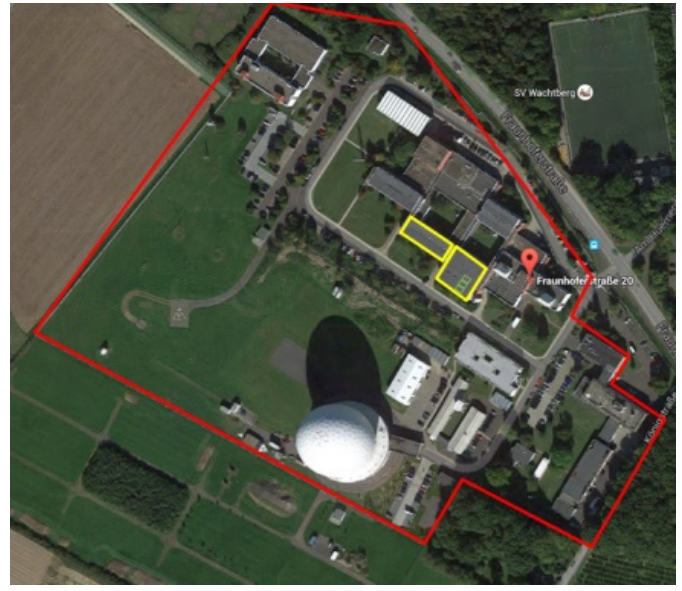

Figure 14: RFID outdoor localization level.
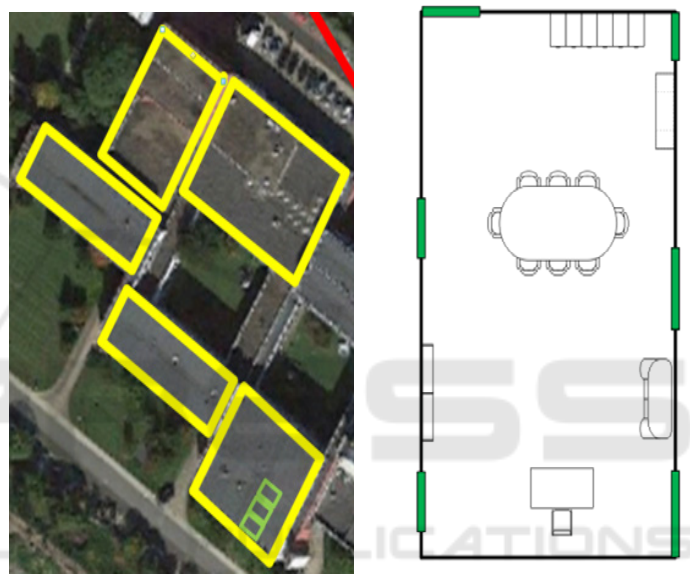

Figure 15: RFID indoor localization level: level 1 (left) and level 2 (right).

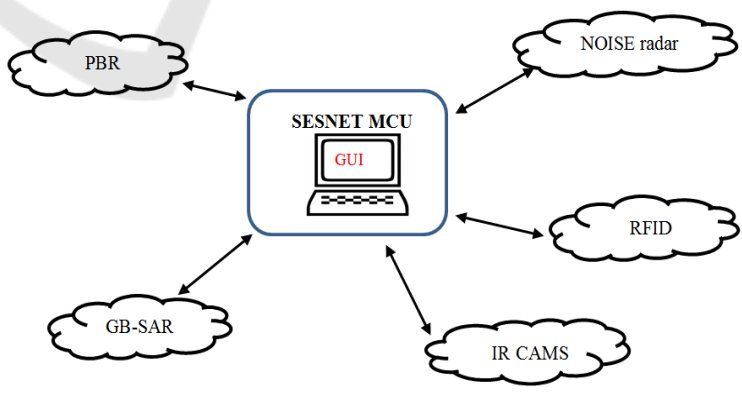

Figure 16: SENSNET communication network basic diagram.

\subsection{Messages Types}

According to the information required or provided by the different nodes, the following types of messages are defined: 
- MCU_Configuration messages: The MCU must be able to modify sensor subsystem operative parameters in order to adapt the SENSNET performance to a changing environment, or to carry out system updates.

- MCU_Management messages: For fulfilling the second level classifier input constrains, S-MCU must be able to ask the IR, the Ku-band

- radar, and the radiometric SAR sub-systems for additional information about specific targets.

- SENSOR_Data Fusion messages: Detection and tracking messages will be generated in each of the nodes belonging to sensor networks responsible of surveillance tasks for target detection and tracking: PBR, NOISE RADAR, Ku-band radar, and RFID. These data will be fed to the data-fusion and/other first classifier stages, following the architecture presented in Figure 6. A common data format must be defined for this type of messages in order to facilitate the data fusion process.

- SENSOR_2LClass messages: As answers to the MCU requests, IR and Ku-band radar subsystems must provide additional information related to detections classified as possible humans.

In order to reduce the required communication network bandwidth, the direct transmission of the signals acquired by each node must be avoided. The use of array antennas and digital beamforming techniques, impose the use of a dedicated data link for each single radiating element of each antenna array, giving rise to an unaffordable transmission bandwidth if raw data is transmitted to the S-MCU or to central nodes designed for the processing of raw data acquired by the nodes of a specific subsystem (sensor network). Local processing also enhances the robustness of the SENSNET, because if one node is attacked, the rest of the nodes will continue working, and could be reconfigured for guaranteeing the physical protection of the infrastructure. So SENSNET will be a distributed sensor network.

Nodes belonging to sensor networks responsible for target detection and tracking will perform detection and tracking tasks and will generate local SENSOR_DATA_Fusion messages that will be sent to the S-MCU, more specifically, to the multisensor detector and tracker. This architecture also improves real time processing capabilities. As an answer to MCU_Management messages, the nodes belonging to sensor networks responsible of providing additional information to the second level classifier, will generate local SENSOR_2LClass messages.

\subsection{Sensors Data Format}

The multisensor detector and tracker module uses the detection and tracking data generated by each sensor that belongs to GB-SAR, PBR, and NOISE radar sensor networks. As additional information, data provided by the RFID network, mainly consisting of identification and locations tags, are also analysed in this data fusion stage in order to improve system surveillance capabilities.

To facilitate the fusion task, a common data format shall be defined for monostatic sensors, as well as for bi/multi-static ones in the Cartesian domain.

In Table 2-Table 6, data fields of the SENSOR_Data_Fusion messages are defined:

- TypeID defines the type of message.

- sourceID identifies the sensor network to which the node belongs to (the type of node).

- rxUid identifies each single node.

- trackID. Each SENSNET node is capable of performing radar observations in either the Range/Doppler or Cartesian plane. By associating consecutive observations the node assigns unique track ID's to target detections.

- timeStamp. All nodes are required to give a time stamp in epoch time, associated to each track ID, making necessary a clock signal distribution among the all SENSET nodes.

To accommodate outputs from many different types of systems, a selection of possible fields is proposed. The column titled Optional indicates fields that might not be strictly needed for the fusion task, or that are redundant if other measurement fields can be provided, indicating for which subsystems that field is required.

IR and Ku-band radar nodes must perform local classification tasks to distinguish between persons, animals and others. SENSOR_2LClass messages will contain the fields defined in Table 2, and specific fields related to image quality and classification accuracy (Table 3) and target features. The final data fields will be defined when the signal processing solutions for classification will be designed. Specific fields could be defined for IR nodes, because the transmission payload associated to IR images is clearly lower than radar ones. 
Table 2: Data exchange format for SENSNET subsystems: target identification fields.

\begin{tabular}{|c|c|c|c|c|}
\hline \multicolumn{4}{|c|}{ Detection Identification fields } \\
\hline 0 & $\begin{array}{c}\text { Field } \\
\text { name }\end{array}$ & Unit & Description & Optional \\
\hline $0 y p$ ID & $\#$ & $\begin{array}{c}\text { MCU_Configuration } \\
\text { (1), } \\
\text { MCU_Management } \\
\text { (2), } \\
\text { SENSOR_Data } \\
\text { Fusion (3), } \\
\text { SENSOR_2LClass } \\
\text { (4),... }\end{array}$ & No \\
\hline 1 & SourceID & $\#$ & $\begin{array}{c}\text { Passive Radar(1), } \\
\text { Noise Radar(2), GB- } \\
\text { SAR(3), RFID(4) }\end{array}$ & No \\
\hline 2 & trackID & $\#$ & $\begin{array}{c}\text { Unique } \\
\text { Identification of the } \\
\text { track }\end{array}$ & No \\
\hline 3 & txUid & $\#$ & $\begin{array}{c}\text { Unique } \\
\text { Identification of } \\
\text { illuminating } \\
\text { transmitter (for } \\
\text { PBR) }\end{array}$ & PBR for \\
\hline 4 & rxUid & $\#$ & $\begin{array}{c}\text { Unique } \\
\text { Identification of the } \\
\text { receiver }\end{array}$ & No \\
\hline 5 & timeStamp & sec & $\begin{array}{c}\text { Unix epoch time } \\
\text { since 1.1.1970 of the } \\
\text { track head }\end{array}$ & No \\
\hline
\end{tabular}

Table 3: Data exchange format for SENSNET subsystems: quality estimation fields.

\begin{tabular}{|c|c|c|c|c|}
\hline \hline \# & $\begin{array}{c}\text { Field } \\
\text { name }\end{array}$ & Unit & Description & Optional \\
\hline 6 & power & $\mathrm{dBW}$ & $\begin{array}{c}\text { Without system } \\
\text { correction } \\
\text { Signal to Noise } \\
\text { ratio of the } \\
\text { latest update }\end{array}$ & Yes \\
\hline 7 & snr_dB & $\mathrm{dB}$ & $\begin{array}{c}\text { Yes } \\
\text { Probability of } \\
\text { this target } \\
\text { track. }\end{array}$ & $\begin{array}{c}\text { No (Yes } \\
\text { for RFID) }\end{array}$ \\
\hline 8 & probability & $\begin{array}{c}\text { Value } \\
\text { from } \\
0.0 \text { to } \\
1.0\end{array}$ & \multicolumn{2}{|c|}{} \\
\hline
\end{tabular}

Table 4: Data exchange format for SENSNET subsystems: estimated target location fields.

\begin{tabular}{|c|c|c|c|c|}
\hline \multicolumn{5}{|c|}{ Targets location fields } \\
\hline 9 & $\begin{array}{c}\text { Field } \\
\text { name }\end{array}$ & Unit & Description & Optional \\
\hline 10 & range & $\mathrm{m}$ & $\begin{array}{c}\text { Monotstatic range to } \\
\text { receiver (rxUid) } \\
\text { Bistatic range with } \\
\text { respect to rxUid and } \\
\text { txUid (for PBR) }\end{array}$ & $\begin{array}{c}\text { For } \\
\text { PBR }\end{array}$ \\
$\begin{array}{c}\text { Not for } \\
\text { PBR }\end{array}$ \\
\hline 11 & Doppler & $\mathrm{Hz}$ & $\begin{array}{c}\text { Bistatic Doppler } \\
\text { with respect to rxUid } \\
\text { and txUid (for PBR) }\end{array}$ & $\begin{array}{c}\text { Not for } \\
\text { PBR }\end{array}$ \\
\hline 12 & azimuth & $\mathrm{deg}$ & $\begin{array}{c}\text { Target azimuth } \\
\text { No }\end{array}$ \\
\hline
\end{tabular}

\begin{tabular}{|c|c|c|c|c|}
\hline & & & $\begin{array}{l}\text { angle receiver with } \\
\text { respect to } 0^{\circ} \text { as true } \\
\text { north (rxUid) }\end{array}$ & \\
\hline 13 & elevation & deg & $\begin{array}{l}\text { Target elevation } \\
\text { angle receiver with } \\
\text { respect to (rxUid). } \\
\text { Horizont at } 0^{\circ} \text {. }\end{array}$ & Yes \\
\hline 14 & latitude & deg & $\begin{array}{c}\text { Target WGS84 } \\
\text { latitude with respect }\end{array}$ & Yes \\
\hline 15 & longitude & deg & $\begin{array}{l}\text { Target WGS84 } \\
\text { longitude with } \\
\text { respect }\end{array}$ & Yes \\
\hline 16 & altitude & $\mathrm{m}$ & $\begin{array}{l}\text { Target altitude } \\
\text { above sea level }\end{array}$ & Yes \\
\hline
\end{tabular}

Table 5: Data exchange format for SENSNET subsystems: estimated target dynamic fields.

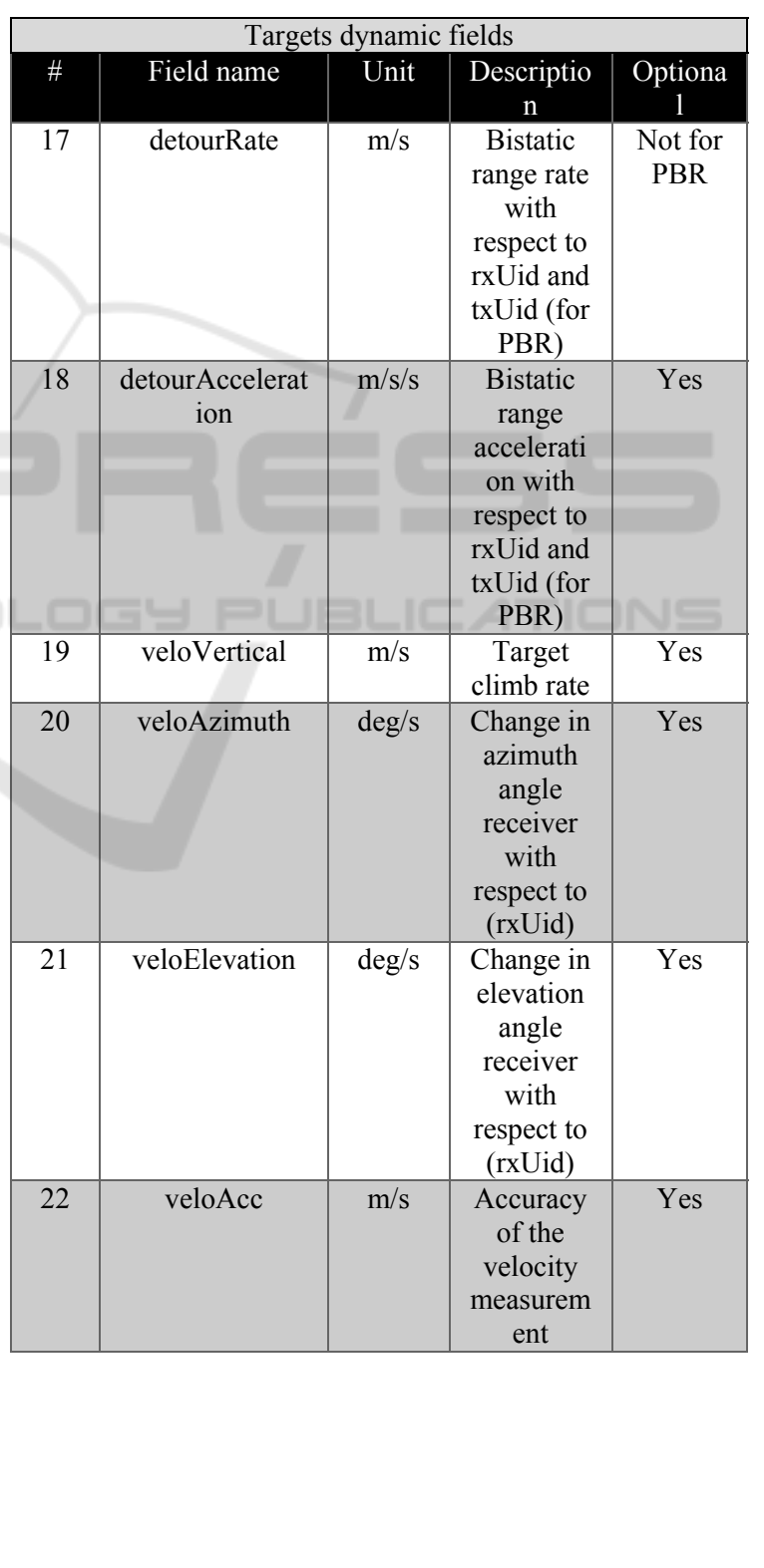


Table 6: Data exchange format for SENSNET subsystems: tracking and plot fields.

\begin{tabular}{|c|c|c|c|c|}
\hline \multicolumn{5}{|c|}{ Tracking fields } \\
\hline$\#$ & Field name & Unit & $\begin{array}{c}\text { Descriptio } \\
\mathrm{n}\end{array}$ & $\begin{array}{c}\text { Optio } \\
\text { nal }\end{array}$ \\
\hline 23 & $\begin{array}{l}\text { xyz_1- } \\
\text { xyz_2- } \\
\text { xyz_3 }\end{array}$ & $\begin{array}{l}\mathrm{m} \\
\mathrm{m} \\
\mathrm{m}\end{array}$ & $\begin{array}{l}\text { Measurem } \\
\text { ent vector. } \\
\text { Easting, } \\
\text { Northing } \\
\text { and } \\
\text { Altitude } \\
\text { of } \\
\text { position, } \\
\text { referred to } \\
\text { the } \\
\text { receiver } \\
\text { location. }\end{array}$ & $\begin{array}{c}\text { If } \\
\text { lat/lo } \\
\mathrm{n} \text { is } \\
\text { given }\end{array}$ \\
\hline 24 & $\begin{array}{l}\text { xyzRate_1 } \\
\text { xyzRate_2- } \\
\text { xyzRate_3 }\end{array}$ & $\begin{array}{l}\mathrm{m} / \mathrm{s} \\
\mathrm{m} / \mathrm{s} \\
\mathrm{m} / \mathrm{s}\end{array}$ & $\begin{array}{c}\text { Measurem } \\
\text { ent vector. } \\
\text { Easting, } \\
\text { Northing } \\
\text { and } \\
\text { Altitude } \\
\text { of } \\
\text { velocity, } \\
\text { referred to } \\
\text { the } \\
\text { receiver } \\
\text { location. }\end{array}$ & Yes \\
\hline$\equiv$ & 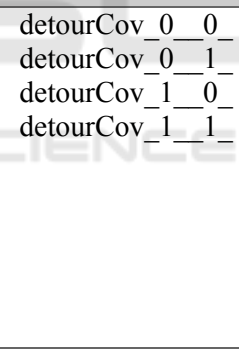 & $\begin{array}{c}\mathrm{m} 2 \\
\mathrm{~m} 2 / \mathrm{s} \\
\mathrm{m} 2 / \mathrm{s} \\
\mathrm{m} 2 / \mathrm{s} 2\end{array}$ & $\begin{array}{c}2 \times 2 \\
\text { Covarianc } \\
\text { e matrix } \\
\text { of the } \\
\text { Range/Do } \\
\text { ppler } \\
\text { track as } \\
\text { detour and } \\
\text { detour } \\
\text { rate }\end{array}$ & $\begin{array}{c}\text { Not } \\
\text { for } \\
\text { PBR }\end{array}$ \\
\hline
\end{tabular}

\subsection{Physical Network}

Given the criticality of the considered scenario, the most reasonable choice, as far as the transmission medium is concerned, is represented by wired connections. Indeed, the use of wireless links would expose SENSNET to attacks, such as jamming, posing serious concerns on the robustness of the system.

Hence, the idea is to rely on Gigabit Ethernet, also considering that such a technology is most probably already deployed in the infrastructure to be protected. It is important to highlight that such a LAN technology, if correctly configured and dimensioned, should be able to guarantee the requested constraints in terms of throughput and delay.

\subsection{Application Layer Protocol}

Regarding the application layer protocol, the idea is to make use of a publish/subscribe framework, able to automatically deal with the real-time nature of the produced data.

In this way, there is no need of implementing "standard" sockets (creating server and clients) for exchanging the collected data, since the communication framework directly manages all the exchanges.

In more detail, the idea is to use the Data Distribution Service for Real-Time Systems (DDS), which is an object management group (OMG) machine-to-machine $(\mathrm{m} 2 \mathrm{~m})$ middleware standard that aims to enable scalable, real-time, interoperable data exchange between publishers and subscribers.

DDS is networking middleware that simplifies complex network programming. It implements a publish/subscribe model for sending and receiving data, events, and commands among the nodes. Nodes that produce information (publishers), in our case the sensors, create "topics" (e.g., location) and publish "samples". DDS delivers the samples to subscribers, in our case the S-MCU, which declare an interest in that topic.

Moreover, DDS allows the user to specify a wide range of parameters for Quality of Service (QoS) support, which make it strongly suitable for realtime communications. Such parameters can be split into four main categories:

- Data availability: decouple applications in time and space. They also enable these applications to cooperate in highly dynamic environments characterized by continuous joining and leaving of publishers and subscribers.

- Data delivery: control the reliability and availability of data, thereby allowing the delivery of the right data to the right place at the right time.

- Data timeliness: provide control over the temporal properties of data.

- Resources: provide control over the local and end-to-end resources, such as memory and network bandwidth.

\subsection{Network Synchronization}

The basic proposed clock distribution scheme is presented in Figure 17. Taking advantage of very low RTT, typical of Gigabit Ethernet based LAN, the trusted and well known Network Time Protocol, NTP, can be used. 
A NTP server could be locally installed in the MCU in order to exploit the infrastructure and the network access deployed for data transmission. Most NTP servers can capture GPS satellite clocks in order to provide a reliable synchronization time signal. Then, using the SENSNET network and the NTP protocol, the time information can be reliably distributed to all nodes.

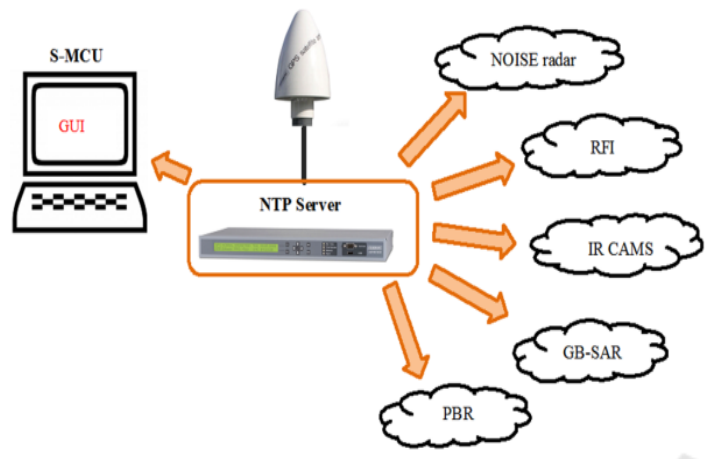

Figure 17: SENSNET synchronization time distribution scheme.

\section{CONCLUSIONS}

The SCOUT system implements the three main security functionalities:

1) Physical attack detection and protection.

2) Cyber-attack detection and countermeasures.

3) Automatic restoration and intelligence reconfiguration of the space control ground station network, in the case of fault of one of the nodes.

Sensor networks are used for acquiring information about potential attacks and/or possible damages of attacks once inflicted. Taking into consideration the different characteristics of physical and cyber intrusions, specific sensor networks are used for monitoring the physical and cyber surveillance environments, respectively.

This paper focusses on the physical surveillance system, describing the main characteristics of the global network of sensor networks exploiting different technologies. Main design drivers were: fault tolerant with respect to light and weather conditions; capability of identifying authorized people and vehicles; intelligent discrimination between people, animals and inanimate objects, preserving the privacy of people; reduce the electromagnetic pollution; low probability of intercept; avoid the requirement of band allocation; reconfigurable, modular, high performance and robust solutions, with low installation and maintenance costs.

The data provided by the sensor networks will be processed by a Main Control Unit (MCU) to determine a situation awareness picture, which is used to assess the degree of alert. If potential threats are detected, the $\mathrm{MCU}$ can order the corresponding sensor network to focus on tracking it.

Each node of each sensor network will perform detection or classification tasks, providing processed data following a defined format. This solution reduces transmission bandwidth requirements, and improves robustness against nodes physical attacks or failures. Considering the security of the communications as a valuable feature for the transmission medium selection, wired networks are in general cheaper and more reliable. Thanks to the low transmission bandwidth, LAN based on Gigabyte Ethernet using UTP cable could be considered.

\section{ACKNOWLEDGEMENTS}

The Optoelectronics Laboratory of the Spanish Aeroespace Institute (INTA) provided a data base of IR images for the design and validation of the infrared sensor network.

This work was partially supported by SCOUT, a research project supported by the European Commission under its 7th Framework Program (contract-no. 607019). The views and conclusions contained herein are those of the authors and should not be interpreted as necessarily representing the official policies or endorsements, either expressed or implied, of the SCOUT project or the European Commission.

\section{REFERENCES}

Moore, R., Lopes, J., 1999. Paper templates. In TEMPLATE'06, 1st International Conference on Template Production. SCITEPRESS.

Smith, J., 1998. The book, The publishing company. London, $2^{\text {nd }}$ edition.

Critical infrastructure protection in the fight against terrorism - COM(2004) 702.

EU Cooperation Theme 10 Security Work Programme 2013. (European Commission c(2013) 3953 of 27 June 2013.

Royal Decree 1066/2001, 28th September, Regulations that define the radio-electric public domain protection conditions, limitations to radio-electric emissions, and 
health protection measures against radio-electric emissions.

ECA Table, The European Table of Frequecy Allocations and Aplications In the frequency range $8.3 \mathrm{kHz}$ to $3000 \mathrm{GHz}$. Approved may 2014.

Mazar, H., International, regional and national regulation of SRDs, ITU WORKSHOP on SHORT RANGE DEVICES (SRDs) AND ULTRA WIDE BAND (UWB). Geneva, 3 June 2014.

IEEE Standard Radar Definitions, IEEE Aerospace and Electronics System Society Sponsored by the Radar System Panel, 2008.

Coleman, C.J., Yardley, H., DAB based passive radar: Performance calculations and trials, International Conference on Radar, 2008, pp. 691-694, September 2008.

Saini, R., Cherniakov, M., DTV signal ambiguity function analysis for radar application, IEE Proceedings on Radar, Sonar and Navigation, vol.152, no.3, pp. 133142, 3 June 2005.

Conti, M., Berizzi, F., Petri, D., Capria A., Martorella, M., High range resolution DVB-T Passive Radar, Radar Conference (EuRAD), 2010 European, Paris, 2010, pp. 109-112.

Kulpa, K., Malanowski, M., Misiurewicz, J., Samczynski, P., Passive radar for strategic object protection, Microwaves, Communications, Antennas and Electronics Systems (COMCAS), 2011 IEEE International Conference on, Tel Aviv, 2011, pp. 1-4.

Gomez-del-Hoyo, P., del-Rey-Maestre, N., Mata-Moya, D., Jarabo-Amores, M.P., First results on ground targets tracking using UHF passive radars under non line-of-sight conditions, Signal Processing Symposium (SPSympo), pp.1-6, June 2015.

Malanowski M., Kulpa, K., Detection of Moving Targets With Continuous-Wave Noise Radar: Theory and Measurements, IEEE Transactions on Geoscience and Remote Sensing, vol. 50, no. 9, pp. 3502-3509, Sept. 2012.

Shelevytsky, I., Kulpa, K., Glushko D., Yanovsky, F.J., Short-range C-band noise radar for meteorological application, Electronics and Nanotechnology (ELNANO), 2013 IEEE XXXIII International Scientific Conference, Kiev, 2013, pp. 473-475.

Lukin, K.A., Mogyla, A.A., Palamarchuk, V.P., Vyplavin, P.L., Zemlyaniy, O.V., Shiyan, Y.A., Zaets, M.K., Ka-band bistatic ground-based noise waveform SAR for short-range applications. Radar, Sonar \& Navigation, IET, 2008, vol. 2, no. 4, pp. 233 243.

Lukin, K.A., Sliding Antennas for Noise Waveform SAR, Applied Radio Electronics, 2005, Vol. 4, \#1, pp. 103106.

Metasensing Airborne SAR, available at: http://www.me tasensing.com/wp/index.php/products/airborne-sar/. Accessed: 15-05-2016.

Marotti, L., Meta, A., Coccia, A., MetaSensing airborne radar: $\mathrm{X}$ - and Ku-band single-pass digital surface model generation, Synthetic Aperture Radar
(APSAR), 2015 IEEE 5th Asia-Pacific Conference on, Singapore, 2015, pp. 184-186.

Vitrociset, A smartphone to navigate the justice of Naples, June 2012. Available at: http://www.vitrociset.it/. Accessed: 15-05-2016. 